\title{
Approches de régénération artificielle de Daniellia oliveri (Rolfe) Hutchison et Dalziel
}

\author{
Rémy HOUEHOUNHA ${ }^{1}$, Hermane T. AVOHOU ${ }^{1 *}$, Brice SINSIN $^{1}$ et \\ André M. TANDJIEKPON ${ }^{2}$ \\ ${ }^{1}$ Laboratoire d'Ecologie Appliquée, Faculté des Sciences Agronomiques, Université d'Abomey-Calavi, 01 BP \\ 526, Cotonou, Bénin. \\ ${ }^{2}$ Centre d'Etudes, de Recherche et de Formations Forestières (CERF-Bénin), 01 BP 526, Cotonou, Bénin. \\ "Auteur correspondant, 08 BP 0982, Cotonou Bénin, Tél. : (229) 21350188, Fax : (229) 21350556, \\ E-mail:h.avohou@cgiar.org,avoher@yahoo.fr,avoher@gmail.com
}

\section{RESUME}

La présente étude teste trois modes de régénération artificielle de Daniellia oliveri par semis de graines et par bouturage de tiges et de racines. Des graines, des boutures de tiges et racines ont été collectées sur des individus de l'espèce puis installées en pépinière sur quatre types de sols. Cinq traitements ont été appliqués aux graines : le témoin, le trempage dans l'eau tiède, dans l'eau chaude, dans l'acide sulfurique et le brûlage. Les résultats ont montré que les graines ont une très bonne aptitude à germer avec des taux de l'ordre de $93 \%$ sans prétraitement. Par contre, tous les prétraitements ont induit des pertes de viabilité. L'espèce peut être aussi reproduite par boutures de racines alors que celles de tiges n'ont pas été viables. Le type de substrat n'influence ni la germination des graines, ni la reprise des bourgeons des boutures. Le traitement des graines par le feu semble stimuler la croissance en hauteur des jeunes pousses sur tous les types de substrats, à l'exception du sol argileux sur lequel le témoin a la meilleure croissance. Globalement, la reproduction par graine présente des taux de réussite et une croissance des jeunes pousses meilleurs aux boutures de racines.

(C) 2009 International Formulae Group. All rights reserved.

Mots clés : Daniellia oliveri, germination, prétraitement, bouturage.

\section{INTRODUCTION}

La dégradation des écosystèmes forestiers en Afrique se produit à un rythme très rapide (FAO, 2001a). Cette dégradation s'accompagne d'un déséquilibre de plus en plus croissant entre la disponibilité des ressources naturelles et les besoins en produits forestiers d'une population sans cesse croissante (Geny et al., 1992 ; FAO, 2001a , 2001b). Il s'avère nécessaire de reconstituer ces écosystèmes non seulement à cause de leurs rôles écologiques et environnementaux importants pour la survie de l'homme, mais aussi pour répondre à la demande croissante des populations en produits forestiers divers
(FAO, 2001a, 2001b). Dans les conditions actuelles d'une forte pression anthropique et d'une dégradation très poussée, la capacité de régénération naturelle et la résilience de ces écosystèmes ne suffisent plus à elles seules pour reconstituer le couvert végétal (Aronson et al., 1993; Bellefontaine et Monteuuis, 2002). L'homme doit alors intervenir par divers processus à savoir la restauration, la réhabilitation ou encore la réallocation (Aronson et al., 1993), en favorisant la régénération naturelle, la multiplication végétative naturelle ou en plantant des espèces d'intérêt (Bellefontaine et al., 2003). Cette intervention de l'homme d'une part nécessite 
une connaissance précise du fonctionnement de ces écosystèmes, de l'écologie et de la biologie des espèces qui les composent et qui pourraient être utilisées dans le processus de reconstitution du couvert végétal, d'autre part exige une maîtrise des modes de régénération naturelle ou artificielle techniquement et économiquement viables et des moyens pour conserver et garantir la disponibilité du matériel génétique de ces espèces (Bellefontaine, 2005).

Pour certaines de ces espèces locales comme Vitellaria paradoxa et Khaya senegalensis, la sylviculture est assez avancée (Robertson, 2002 ; Sokpon et Ouinsavi, 2004). Mais pour la plupart comme Isoberlinia doka, Detarium microcarpum, Daniellia oliveri, Pentadesma butyracea, etc. qui sont aussi d'un grand intérêt socio-économique pour les populations (Bellefontaine et al., 2000), les informations scientifiques et techniques élémentaires pour leur sylviculture ou leur conservation sont encore embryonnaires et le matériel génétique de qualité n'est souvent pas disponible (Bellefontaine et al., 2000 ; Harviel et al., 2006). La faible disponibilité de ces informations entrave toute approche de conservation et de sylviculture (reboisement ou restauration) devant limiter les pertes de la diversité de ces espèces et répondant aux besoins des communautés (Bellefontaine et al., 2000).

La présente étude porte sur Daniellia oliveri (Rolfe) Hutch \& Dalz, une espèce à usage multiple répandue dans les savanes soudaniennes. Divers organes de l'espèce sont utilisés pour leurs propriétés médicinales (Jegede et al., 2006 ; Ahmadua et al., 2007). Les feuilles et les fleurs sont utilisées comme fourrages en zone soudano-sahélienne (Lykke, 2000) et le bois comme matériau de construction et combustible (Lykke, 2000 ; FAO, 2001b). Toutefois, l'espèce se raréfie de plus en plus dans certaines zones sahéliennes (Lykke, 2000).

L'étude vise à jeter les bases de la sylviculture de $D$. oliveri. De manière spécifique, il s'agit de tester et comparer sur divers substrats, trois techniques de multiplication artificielle de l'espèce à savoir le semis de graines ayant subi divers prétraitements germinatifs, les boutures de tiges et les boutures de racines, du point de vue des taux de germination ou de réussite du bouturage et de la croissance des plantules issues des divers types de matériel végétal.

\section{MATERIEL ET METHODES \\ Matériel végétal}

Daniellia oliveri (Rolfe) Hutch \& Dalz est distribué du Sénégal au Cameroun. C'est une mésophanérophyte de la famille des Légumineuses et de la sous-famille des Caesalpiniaceae. Arbre des savanes soudaniennes et guinéennes pouvant atteindre une hauteur de $30 \mathrm{~m}$, il possède une cime dense facilement reconnaissable par sa couronne en forme de cône renversé (Arbonier, 2000). Son fût est de couleur blanc grisâtre, droit et souvent bas-branchu. Ses feuilles sont paripennées et les fleurs sont en panicules de couleur blanche et longues de 15 à $30 \mathrm{~cm}$. Le fruit est une gousse lisse, plate et déhiscente à maturité. Il contient une graine légère, obovale et longue de 15 à $20 \mathrm{~mm}$. Elle est facilement projetée par le vent sur de longues distances pour ensemencer champs et jachères avoisinants (Cuny et al., 1997). Mais l'espèce se propage aussi facilement par voie asexuée (Bellefontaine, 1997).

Dans le cadre de cet essai, trois types de matériel végétal de reproduction ont été collectés sur des individus d'une même population: les graines à maturité, les boutures de tiges et les boutures de racines. Les boutures de racines sont des morceaux de racines latérales de $20 \mathrm{~cm}$ de long prélevés à une profondeur de 10 à $20 \mathrm{~cm}$ du sol. Les boutures de tiges sont des segments de $20 \mathrm{~cm}$ de long prélevés sur de jeunes rameaux aoûtés âgés d'environ trois ans.

\section{Zone d'étude}

Les essais ont été réalisés au Centre du Bénin entre $7^{\circ} 10^{\prime}$ et $8^{\circ} 47^{\prime}$ latitude Nord et $1^{\circ} 36^{\prime}$ et $2^{\circ} 43^{\prime}$ longitude Est (figure 1). La région bénéficie d'un climat de transition entre le climat subéquatorial de la côte et le climat tropical humide de type soudanoguinéen du Nord-Bénin. La pluviométrie moyenne annuelle varie entre 900 et 1200 $\mathrm{mm}$. Les températures moyennes mensuelles sont comprises entre $20{ }^{\circ} \mathrm{C}$ et $25{ }^{\circ} \mathrm{C}$ et les écarts journaliers sont faibles. Les sols 


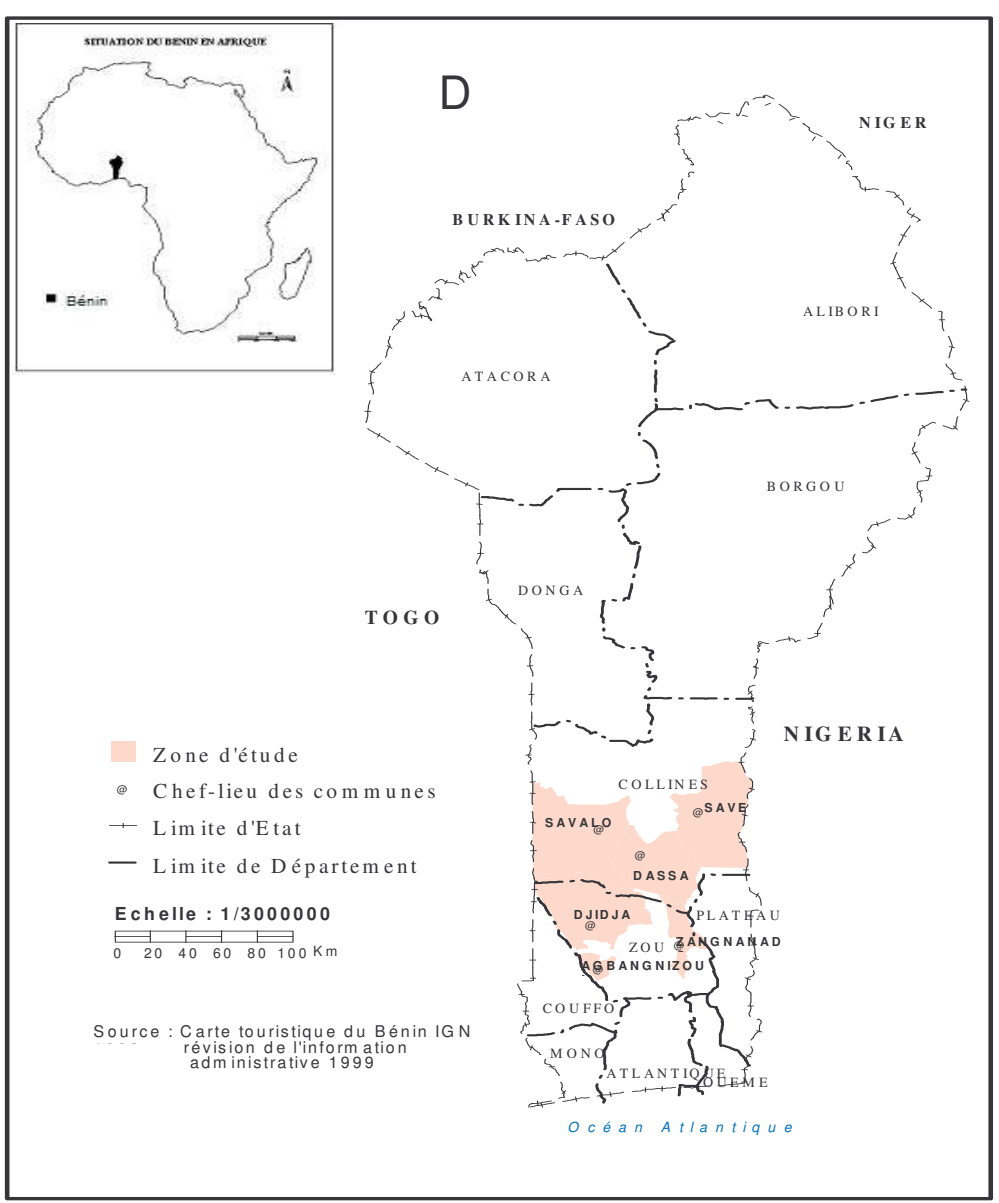

Figure 1: Carte de localisation de la zone d'étude.

comprennent des sols ferralitiques dans la partie Sud de la zone d'étude et des sols ferrugineux à texture variable dans la partie Nord.

\section{Dispositif expérimental, collecte et analyse des données}

Des tests de germination de graines et de reprise des bourgeons de tiges et de racines ont été conduits en station. Une étude de la croissance des plantules issues des trois types de matériel végétal a été aussi réalisée.

Pour les tests de germination, les graines après extraction des gousses, ont été réparties en cinq lots. Quatre des cinq lots ont subi chacun un type de prétraitement proposé par Ffolliott et Thames (1983) pour les semences forestières et le dernier lot a été gardé comme témoin. Cinq traitements de semences ont été donc considérés et se présentent comme suit :

(i) sans prétraitement,

(ii) trempage dans l'eau ordinaire pendant 24 $\mathrm{h}$,

(iii) trempage dans l'eau chaude jusqu'à refroidissement,

(iv) trempage dans l'acide sulfurique, et

(v) brûlage avec la paille sèche.

Toutes les graines sont semées à plat, c'est-àdire dans la position qu'elles adoptent en tombant des arbres.

Pour les tests de bouturage, les boutures n'ont subi aucun prétraitement. Elles ont été obliquement plantées dans le substrat. Pour éviter la pourriture précoce des boutures, le bout aérien de chaque bouture est taillé en 
biais et protégé par une toile en plastique afin d'empêcher l'infiltration des eaux de pluie ou d'arrosage dans les tissus.

Quatre types de substrat représentant les principaux types de sol de la zone ont été utilisés afin de déterminer l'influence du sol sur la germination ou sur la reprise des bourgeons et sur la croissance des plantules. Il s'agit du sol ferrugineux argileux, du sol ferrugineux sablonneux, du sol ferralitique et du sol latéritique. Les substrats ferralitiques et latéritiques sont acides, très dégradés avec une faible teneur en matières organiques (tableau 1). Les substrats ferrugineux argileux et sablonneux par contre sont neutres, moins dégradés avec une teneur en matières organiques plus élevée (tableau 1).

Le dispositif expérimental adopté aussi bien pour les tests de germination que pour les tests de reprise des bourgeons est en blocs aléatoires complets à deux facteurs et à deux répétitions. Les facteurs sont d'une part le type de substrat et le type de prétraitement pour les tests de germination et d'autre part le type de substrat et le type de bouture pour les tests de bouturage. Pour chaque répétition, 50 graines ont été semées dans le cas des tests de germination et 50 boutures ont été plantées pour les tests de bouturage.

Les données suivantes ont été soit journellement ou mensuellement collectées :

(i) le nombre de plantules levées et le nombre de boutures de tiges ou de racines bourgeonnées sont notés en 30 jours, du 25 janvier 2007 au 24 février 2007 ; (ii) la hauteur de 10 pousses au niveau de chaque traitement a été mesurée mensuellement à partir du premier mois jusqu'au cinquième mois (24 février $2007 \mathrm{au}$ 25 juillet 2007).

Des tests d'analyse de variance ont été réalisés avec un seuil de signification de 5\% pour :

(i) d'une part déterminer l'effet des prétraitements et du substrat sur la germination des graines, et l'effet du type bouture et du substrat sur le taux de reprise des bourgeons, et d'autre part comparer les taux de germination des graines aux taux de reprise des bougeons : Dans chacun de ces cas, le modèle utilisé est un modèle croisé fixe avec comme facteurs, le prétraitement et le substrat ou le type de bouture et le substrat,

(ii) En outre, déterminer l'effet des prétraitements et du substrat sur la croissance des plantules issues des graines, et l'effet du type bouture et du substrat sur la croissance des pousses issues des boutures, et aussi comparer les performances de croissance des plantules issues des graines et des pousses issues de boutures sur différents types de substrat: Dans chacun des cas un modèle d'analyse de variance avec mesures répétées a été utilisé, le facteur de mesures répétées étant le facteur temps avec cinq niveaux correspondant aux cinq dates de mesure des hauteurs.

La normalité des résidus a été vérifiée grâce au test de Shapiro-Wilk et l'homogénéité des variances grâce au test de Levene (Zar, 1999). Deux types de

Tableau 1 : Propriétés physico-chimiques des substrats utilisés pour la multiplication artificielle de D. oliveri en pépinière.

\begin{tabular}{|c|c|c|c|c|}
\hline \multirow{2}{*}{$\begin{array}{c}\text { Paramètres physico- } \\
\text { chimiques }\end{array}$} & \multicolumn{4}{|c|}{ Substrats } \\
\hline & $\begin{array}{c}\text { Sol ferrugineux } \\
\text { argileux }\end{array}$ & $\begin{array}{c}\text { Sol ferrugineux } \\
\text { sablonneux }\end{array}$ & $\begin{array}{c}\text { Sol } \\
\text { ferrallitique }\end{array}$ & Sol latéritique \\
\hline $\mathrm{C}(\%)$ & 2,94 & 1,79 & 0,16 & 0,27 \\
\hline $\mathrm{C} / \mathrm{N}$ & 16,30 & 12,00 & 2,30 & 4,50 \\
\hline M.O. (\%) & 5,06 & 3,08 & 0,27 & 0,46 \\
\hline $\mathrm{pH}_{\mathrm{eau}}(1 / 2,5)$ & 7,20 & 7,40 & 6,40 & 5,90 \\
\hline $\mathrm{pH}_{\mathrm{kcl}}(1 / 2,5)$ & 6,70 & 6,90 & 5,10 & 5,00 \\
\hline $\mathrm{K}^{+}$éch. (Méq/100g) & 0,36 & 0,46 & 0,05 & 0,16 \\
\hline $\mathrm{Na}^{+}$éch. (Méq/100g) & 0,08 & 0,06 & 0,04 & 0,06 \\
\hline CEC (Méq/100g) & 17,50 & 9,80 & 10,20 & 8,30 \\
\hline
\end{tabular}

C/N : Rapport $\mathrm{C} / \mathrm{N}, \mathrm{K}^{+}$éch. : Ions potassium échangeables, $\mathrm{Na}^{+}$éch. : Ions sodium échangeables, CEC : Capacité d'échange cationique, M.O. : Teneur en matière organique, $\mathrm{C}:$ Teneur en Carbone. 
transformation de variables ont été effectués dans le cas où les conditions d'applicabilité de l'analyse de variance n'étaient pas remplies : (i) la transformation angulaire pour les taux de germination ou de reprise des bourgeons par la formule $y=2 \arcsin \sqrt{x}$ où x représente les taux de germination ou de reprise des bourgeons en pourcentage, (ii) la transformation logarithmique par la formule $y=10 \log x$ où $\mathrm{x}$ représente la hauteur des plantules. Dans le cas de différences significatives, le test de Student-NewmanKeuls a été utilisé pour la séparation des moyennes (Zar, 1999).

Pour les modèles d'analyse de variance avec mesures répétées, l'hypothèse de sphéricité a été vérifiée par le test de Mauchly et dans le cas de violation de l'hypothèse, l'ajustement de Huynh-Feldt avec les $F$ et les valeurs de probabilité associées ont été utilisés.

\section{RESULTATS}

Effet des prétraitements germinatifs et du substrat sur la germination des graines

Le tableau 2 présente les taux de germination des graines par prétraitement et par substrat. L'interaction entre le prétraitement et le type de substrat n'est pas significative $(\mathrm{F}=0,4 ; \mathrm{p}=0,960)$. Le type de substrat n'influence pas la germination des graines $(F=1,1 ; p=0,356)$. Globalement, les taux moyens de germination par substrat fluctuent entre $63,0 \pm 29,8 \%$ et $66,0 \pm 30,2 \%$ tous types de prétraitements confondus. Le prétraitement germinatif par contre influe sur la germination des graines $(\mathrm{F}=239,8 ; \mathrm{p}=$ $0,000)$. Les graines non traitées présentent le taux de germination le plus élevé $(93,0 \pm$ $3,4 \%)$. Elles sont suivies des graines prétraitées à l'acide sulfurique $(85,0 \pm 4,3 \%)$, des graines trempées dans l'eau simple (70,0 \pm $6,5 \%)$. Les graines trempées dans l'eau chaude et les graines traitées au feu ont les taux de germination les plus faibles (respectivement 58,0 $\pm 5,0 \%$ et $13,8 \pm 2,0 \%$ ).

Effet du type de bouture et du substrat sur les taux de reprise des bourgeons en 30 jours

Le tableau 3 présente les taux de reprise des bourgeons des boutures de racines et de tiges pour les quatre types de substrat après 30 jours. Le type de substrat n'influe pas significativement sur la reprise des bourgeons des boutures $(\mathrm{F}=2,1 ; \mathrm{p}=0,176)$. Par contre, le taux de reprise des bourgeons varie significativement en fonction du type de bouture $(F=210,8 ; p=0,000)$. Les boutures

Tableau 2: Taux moyens de germination (\%) des graines de D. oliveri pour cinq types de prétraitement et quatre types de substrat.

\begin{tabular}{lccccc}
\hline Prétraitements & \multicolumn{4}{c}{ Substrats } & Moyenne \\
\cline { 2 - 5 } & $\begin{array}{c}\text { Sol } \\
\text { ferrugineux } \\
\text { sablonneux }\end{array}$ & $\begin{array}{c}\text { Sol } \\
\text { ferrallitique }\end{array}$ & $\begin{array}{c}\text { Sol } \\
\text { latéritique }\end{array}$ & $\begin{array}{c}\text { Sol } \\
\text { ferrugineux } \\
\text { argileux }\end{array}$ & \\
\hline Aucun & $93,0 \pm 4,2$ & $94,0 \pm 2,8$ & $93,0 \pm 1,4$ & $93,0 \pm 7,1$ & $93,0 \pm 3,4 \mathbf{a}$ \\
Eau & $68,0 \pm 11,3$ & $69,0 \pm 1,4$ & $55,0 \pm 4,2$ & $77,0 \pm 4,2$ & $70,0 \pm 6,5 \mathbf{c}$ \\
Eau chaude & $60,0 \pm 5,4$ & $60,0 \pm 8,5$ & $55,0 \pm 4,2$ & $57,0 \pm 4,2$ & $58,0 \pm 5,0 \mathbf{d}$ \\
Acide & $82,0 \pm 5,4$ & $84,0 \pm 2,8$ & $85,0 \pm 4,2$ & $89,0 \pm 4,2$ & $85,0 \pm 4,3 \mathbf{b}$ \\
Brûlage & $12,0 \pm 2,8$ & $15,0 \pm 1,4$ & $13,0 \pm 1,4$ & $15,0 \pm 1,4$ & $13,7 \pm 2,0 \mathbf{e}$ \\
\hline
\end{tabular}

\begin{tabular}{lcccc}
\hline Moyenne $63,0 \pm 29,8 \mathbf{a}$ & $64,0 \pm 29,0 \mathbf{a}$ & $63,0 \pm 29,8 \mathbf{a}$ & $66,0 \pm 30,2 \mathbf{a}$ & - \\
\hline Substrat : $\mathrm{F}=1,1, \mathrm{p}=0,356 ;$ prétraitement $: \mathrm{F}=239,8, \mathrm{p}=0,000 ;$ Substrat* ${ }^{*}$ prétraitement : $\mathrm{F}=0,370, \mathrm{p}=0,960$. Les
\end{tabular} moyennes suivies de la même lettre ne sont pas statistiquement différentes suivant le test de Student-Newman Keuls au seuil de $5 \%$. 
Tableau 3: Taux moyen de reprise des bourgeons racine et de tige de $D$. oliveri pour quatre types de substrat.

\begin{tabular}{lcc}
\hline \multirow{2}{*}{ Substrats } & \multicolumn{2}{c}{ Taux de reprise des bourgeons } \\
\cline { 2 - 3 } & Boutures de racines & Boutures de tiges \\
\hline Sol ferrugineux sablonneux & $70,0 \pm 2,8$ & $41,0 \pm 4,2$ \\
Sol ferralitique & $62,0 \pm 2,8$ & $37,0 \pm 4,2$ \\
Sol latéritique & $66,0 \pm 0,0$ & $41,0 \pm 7,01$ \\
Sol ferrugineux argileux & $66,0 \pm 0,0$ & $36,0 \pm 2,8$ \\
\hline Moyenne & $66,0 \pm 3,4$ & $38,8 \pm 4,4$ \\
\hline Substrat : $\mathrm{F}=2,1, \mathrm{p}=0,176 ;$ Type de bouture : $\mathrm{F}=210,8 ; \mathrm{p}=0,000 ;$ substrat*type de bouture : \\
$\mathrm{F}=0,5, \mathrm{p}=0,680$.
\end{tabular}

de racines présentent les taux de reprise les plus élevés $(66,0 \pm 3,4 \%)$ et les boutures de tiges les taux de reprise les plus faibles $(38,8$ $\pm 4,4 \%)$.

\section{Croissance en pépinière des plantules issues des différentes graines prétraitées pour quatre types de substrat}

Pour l'ensemble des traitements et des substrats, la hauteur des plantules varie significativement entre 30 et 150 jours $(F=$ $3824,5 ; \mathrm{p}=0,000)$. Toutefois, le rythme de croissance est influencé par le prétraitement des graines $(\mathrm{F}=87,2 ; \mathrm{p}=0,000)$ et le type de substrat $(\mathrm{F}=51,9 ; \mathrm{p}=0,000)$.

Les figures $2 \mathrm{a}$ à $2 \mathrm{~d}$ comparent la croissance des plantules issues des cinq types de graines prétraitées sur chacun des quatre différents substrats.

Sur les sols sablonneux et ferralitique (figure $2 \mathrm{a}$ et $2 \mathrm{~b}$ ), la hauteur des plantules croît rapidement pour tous les types de prétraitements, mais les plantules issues des graines prétraitées avec le feu montrent les hauteurs les plus élevées sur les 150 jours. Elles sont suivies par les plantules issues des graines traitées à l'acide sulfurique et dans une moindre mesure les plantules issues des graines trempées dans l'eau simple. Les plantules issues des graines non traitées et trempées dans l'eau chaude montrent des hauteurs les plus faibles après 90 jours.

Sur les sols latéritiques (figure 2c), bien que les plantules issues des graines brûlées montrent les hauteurs les plus élevées pendant les trois premiers mois $(F=292,7$ et $p=0,000$ au mois $1 ; \mathrm{F}=351,1$ et $\mathrm{p}=0,000$ au mois 2 ; $\mathrm{F}=128,2$ et $\mathrm{p}=0,000$ au mois 3 ), leur vitesse de croissance sur les 150 jours est plus faible que les autres prétraitements. Les plantules issues des graines traitées à l'acide sulfurique, bien qu'ayant une hauteur initiale moyenne faible, croissent rapidement entre 30 et 90 jours. A partir de 120 jours on observe plus de différences significatives entre les hauteurs de ces plantules et celles des plantules issues des graines brûlées. Les plantules issues des graines non traitées, trempées dans l'eau simple ou dans l'eau chaude montrent des hauteurs significativement inférieures aux deux autres sur les 150 jours. Par ailleurs à partir de 90 jours leur rythme de croissance diminue, à l'exception des plantules issues des graines non traitées pour lesquelles la croissance s'accélère et augmente significativement à 150 jours.

Sur le sol argileux (figure 2d), les plantules issues des graines brûlées montrent les hauteurs les plus élevées pendant les trois premiers mois $(\mathrm{F}=659,4$ et $\mathrm{p}=0,000$ au mois $1 ; \mathrm{F}=561,6$ et $\mathrm{p}=0,000$ à deux mois ; $\mathrm{F}=$ 210,8 et $p=0,000$ et à trois mois), bien que leur rythme de croissance sur les 150 jours soit plus faible que les autres prétraitements dont les plantules croissent très rapidement. A partir de 120 jours, ce sont plutôt les plantules issues des graines non traitées qui présentent les hauteurs les plus élevées, suivies des graines traitées au feu et à l'acide sulfurique $(\mathrm{F}=126,4$ et $\mathrm{p}=0,000$ à 120 jours $; \mathrm{F}=128,9$ et $\mathrm{p}=0,000$ à 150 jours). 
(a)

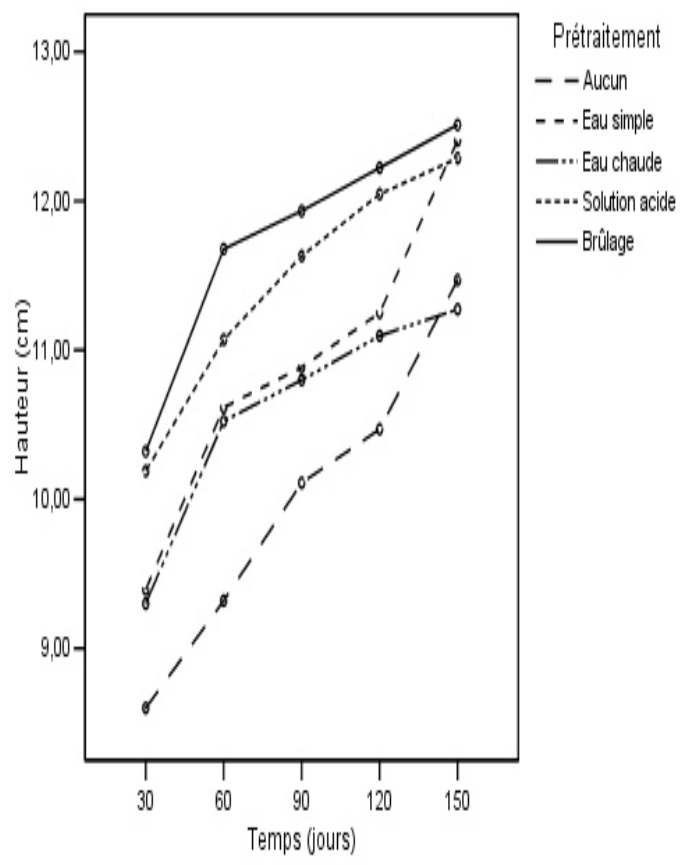

(c)

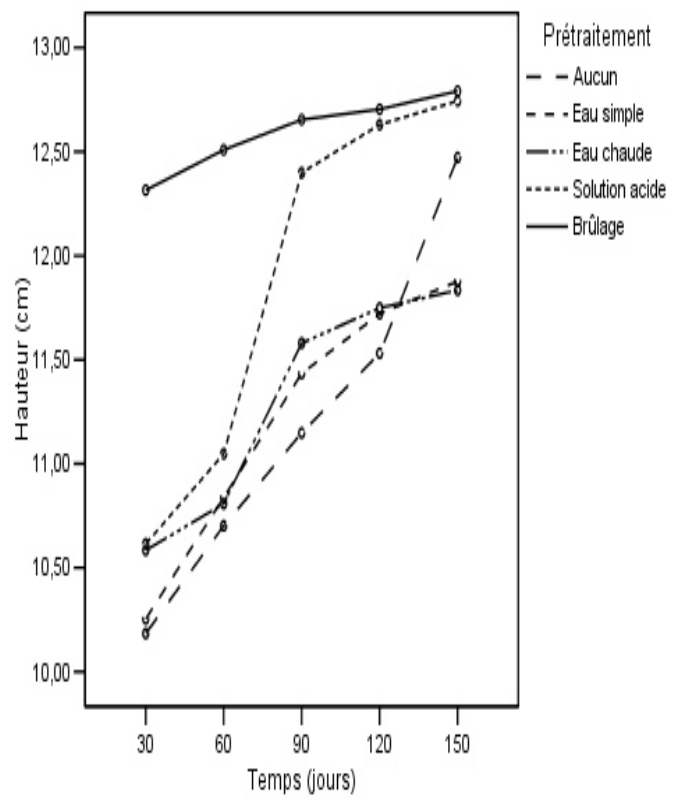

(b)

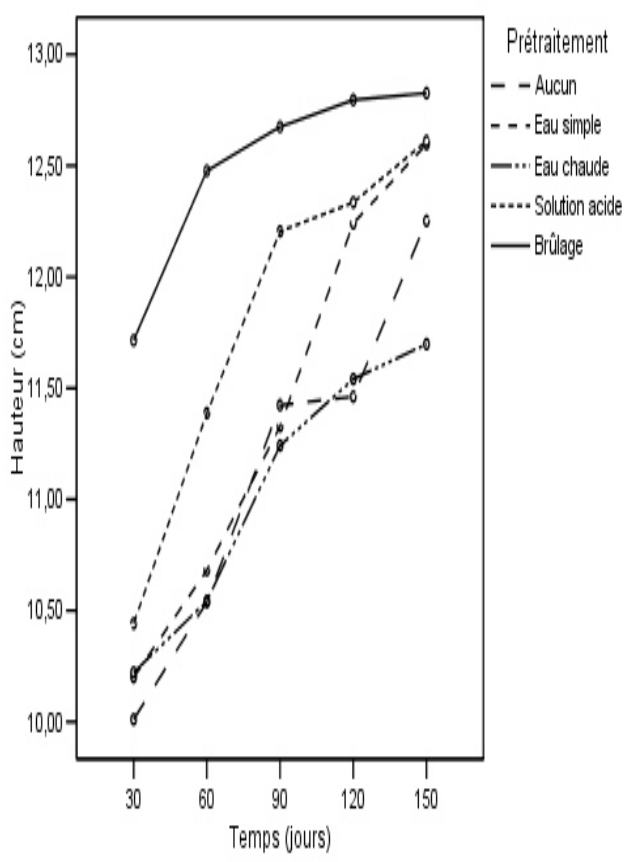

(d)

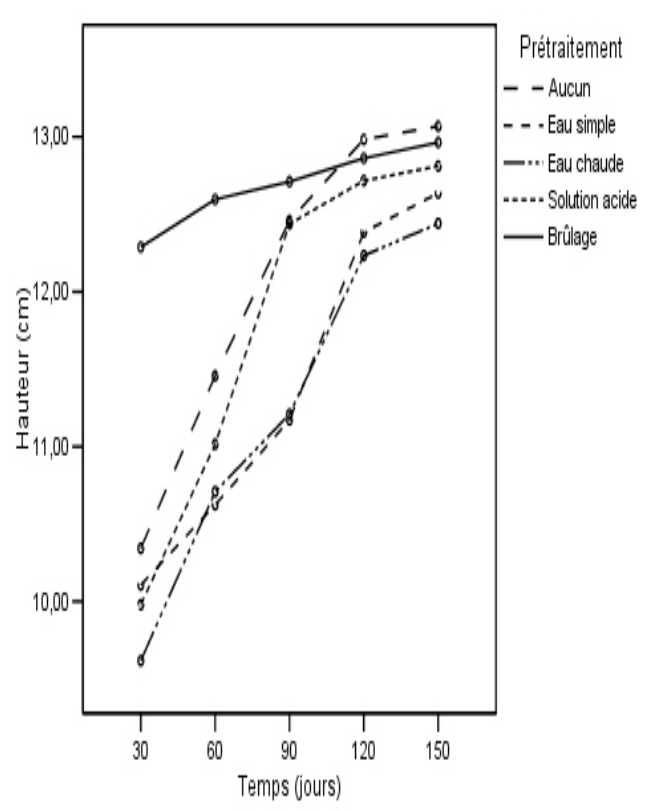

Figure 2: Croissance des plantules issues des cinq types de graines sur quatre différents substrats. (a) Sol ferrugineux sablonneux, (b) Sol ferralitique, (c) Sol latéritique, (d) Sol ferrugineux argileux. 
En outre, la vitesse de croissance des plantules au niveau de tous les traitements diminue à partir du quatrième mois. Les plantules issues des graines traitées à l'eau chaude et à l'eau simple présentent les hauteurs les plus faibles sur les cinq mois.

En général, c'est le sol argileux qui favorise les croissances les plus significatives, suivie par le sol latéritique et le sol ferralitique. Les plus faibles croissances sont observées au niveau du sol sablonneux ( $\mathrm{F}=$ $284,3 ; \mathrm{p}=0,000)$.

Croissance des plantules issues des boutures de tiges et de racines sur quatre types de substrat.

Les figures $3 \mathrm{a}$ et $4 \mathrm{~b}$ présentent la croissance des pousses issues de boutures de tiges et de racines sur les quatre différents types de substrat. La hauteur des pousses varie significativement avec le temps ( $\mathrm{F}=10129,5$; $p=0,000)$. Toutefois, cette variation est significativement influencée par le type de bouture $(\mathrm{F}=31587,3 ; \mathrm{p}=0,000)$ et le type de substrat. $(\mathrm{F}=1278,4 ; \mathrm{p}=0,000)$.
Les pousses issues des boutures de tiges ont une hauteur moyenne en 30 jours très faible, elle est inférieure à $3 \mathrm{~cm}$ quelque soit le type de substrat (figure 3a). On note une chute brutale de la hauteur des plantules entre 30 et 60 jours et une mortalité totale de toutes les pousses pour tous les types de sol excepté le sol sablonneux. Pour ce dernier, la hauteur des pousses croît légèrement entre 30 et 60 jours et chute aussi brutalement aboutissant à une mortalité totale des plantules à 90 jours.

Les plantules issues des boutures de racines ont une hauteur à 30 jours significativement supérieure à celle des plantules issues des boutures de tiges. Pour tous les types de substrat, la hauteur augmente significativement avec le temps (figure $3 b$ ). Pendant les deux premiers mois, les hauteurs les plus élevées sont obtenues sur le sol argileux $(F=299,4$ et $p=0,000$ à un mois ; $F$ $=49,5$ et $p=0,000$ à deux mois). A partir de trois mois, les hauteurs les plus élevées sont obtenues sur le sol latéritique suivi du sol argileux $(\mathrm{F}=223,9$ et $\mathrm{p}=0,000$ à trois mois ; $\mathrm{F}=184,3$ et $\mathrm{p}=0,000$ à quatre mois $; \mathrm{F}=$ 111,6 et $\mathrm{p}=0,000$ à cinq mois). (a)

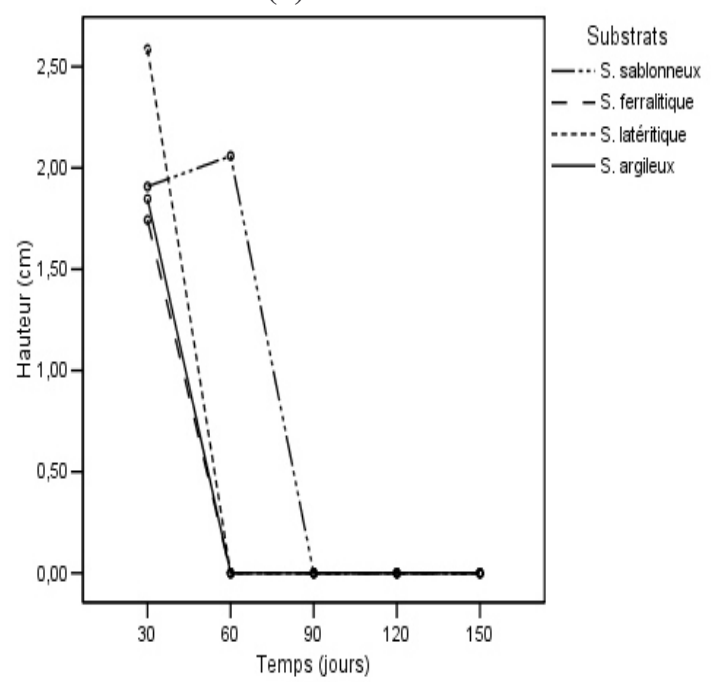

(b)

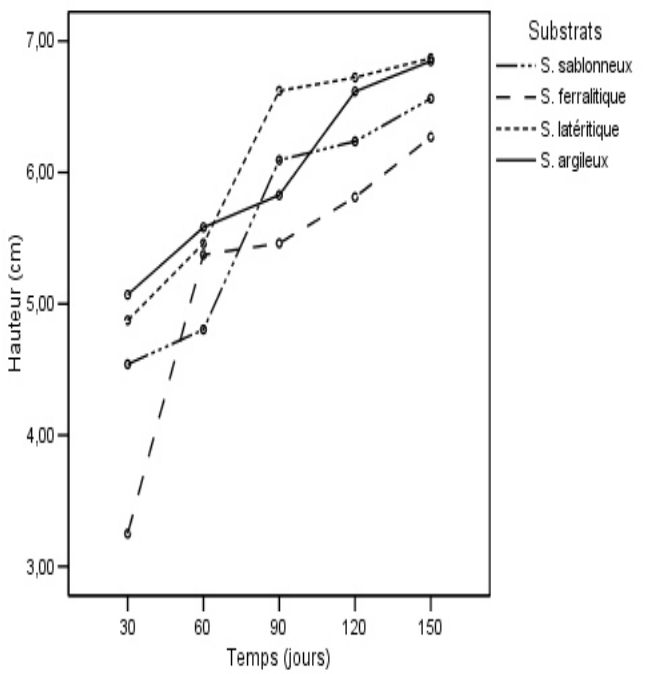

Figure 3: Croissance des plantules issues des boutures de tiges et de racines sur quatre types de substrat. (a) Plantules issues des boutures de tiges, (b) Plantules issues des boutures de racines. S. sablonneux : sol ferrugineux sablonneux ; S. ferralitique : sol ferralitique ; S. latéritique : sol latéritique ; S. argileux : sol ferrugineux argileux. 
Comparaison des taux de réussite et de la croissance des pousses issues des graines et des boutures de racines

La figure 4 compare pour les quatre types de substrat, les taux de germination des graines prétraitées aux taux de reprise des bourgeons des boutures de racines. Le taux de réussite varie significativement en fonction de la nature du matériel végétal utilisé $(\mathrm{F}=$ $225,5 ; \mathrm{p}=0,000)$. Les graines non traitées présentent le taux de réussite le plus élevé $(93,2 \pm 3,4 \%)$. Elles sont suivies des graines traiées à l'acide sulfuriques $(85,0 \pm 4,3 \%)$. Les boutures de racines présentent des taux de réussite qui ne sont pas significativement différents des graines trempées dans l'eau simple (respectivement $66,0 \pm 3,4 \%$ et $70,3 \pm$ $6,5 \%$ ). Les graines préalablement brûlées ou trempées dans l'eau chaude présentent les taux de réussite les plus faibles (respectivement $13,7 \pm 2,0 \%$ et $58,0 \pm 5,0 \%$ ).

La figure 5 compare la croissance des plantules issues des graines à la croissance des jeunes pousses issues des bourgeons des boutures de racines. De façon générale, les pousses issues des boutures de racines montrent des hauteurs significativement inférieures à celles des plantules issues des graines quels que soient les types de prétraitement appliqués $(\mathrm{F}=15225,2$; $\mathrm{p}=$ 0,000). La hauteur moyenne des pousses issues des boutures de racines fluctue entre $4,4 \pm 0,7 \mathrm{~cm}$ à un mois et $6,6 \pm 0,3 \mathrm{~cm}$ à cinq mois. Pour les plantules issues des graines, elle varie en moyenne entre $9,3 \pm 2,4 \mathrm{~cm}$ le premier mois et $11,4 \pm 2,2 \mathrm{~cm}$ le cinquième mois.

\section{DISCUSSION}

La reconstitution du couvert végétal dans les programmes d'aménagement forestier peut se faire par semis, plantation, rejet de souches après recépage, marcottage naturel et drageonnage (Bellefontaine et al., 2003). Il convient d'analyser les options techniquement et économiquement viables en fonction des espèces concernées et des moyens disponibles (Bellefontaine, 2005). D. oliveri se reproduit par voie sexuée (Cuny et al., 1997 ; Ouédraogo et al., 2003) et il a été rapporté que l'espèce produit abondamment de semences

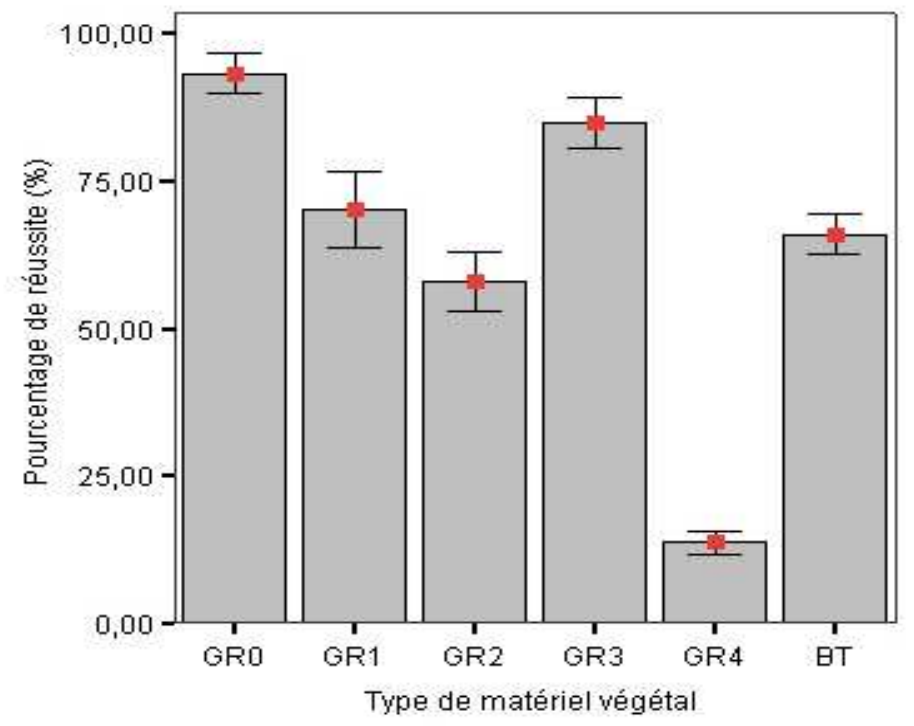

Figure 4: Comparaison des taux de réussites à 30 jours des modes de propagation par graines et par boutures de segments de racines. GR0: Graines non traitées, GR1: Graines trempées dans l'eau simple, GR2: Graines trempées dans l'eau chaude, GR3: Graines traitées à l'acide sulfurique, GR4: Grainées traitées au feu, BT: Boutures de racine. Nature du matériel végétal: $\mathrm{F}=225,51, \mathrm{p}=0,000$; Substrat : $\mathrm{F}=0,98 ; \mathrm{p}=0,418$; interaction type de matériel végétal*substrat : $\mathrm{F}=0,55 ; \mathrm{p}=0,884$. Les barres d'erreur représentent les déviations standard. 
(Cuny et al., 1997). Il se reproduit également par voie asexuée (Bellefontaine, 1997 ; Harivel et al., 2006). Particulièrement, les modes de reproduction végétative par drageons ou rejets de souche ont été mentionnés (Bellefontaine, 1997 ; Harivel et al., 2006). L'étude a permis d'explorer les possibilités d'utilisation de divers modes de reproduction sexuée et asexuée à savoir le semis de graines et les boutures de tiges et de racines, dans la multiplication artificielle de $D$. oliveri en général et la production de plants en pépinière en particulier. Les résultats ont montré que les graines de $D$. oliveri ont une très bonne aptitude à la germination. Elles ne nécessitent pas de prétraitements ou un type de substrat particulier. Les graines mûres de l'espèce ne souffrent donc pas de problèmes de dormance. Toutes les méthodes de prétraitement testées dans le cadre de cette étude ont induit des pertes de viabilité des semences, avec des effets variables suivant les méthodes. Le prétraitement qui affecte le moins la viabilité des graines est le trempage dans une solution d'acide sulfurique avec des pertes de l'ordre de 9-10\% par rapport aux graines non-traitées. Mais les traitements qui affectent le plus la viabilité des graines sont le brûlage au feu et le trempage dans l'eau chaude avec des pertes de l'ordre de $80 \%$ pour le brûlage et $35 \%$ pour l'eau chaude. La chaleur semble donc un facteur très préjudiciable à la viabilité des graines de $D$. oliveri.

Outre, leur bonne aptitude à la germination, les graines de $D$. oliveri ont aussi une bonne aptitude à la conservation dans les conditions paysannes (Ouedraogo et al., 2003). En effet, elles peuvent se conserver pendant plus d'un an avec les moyens traditionnels peu coûteux et sans une perte significative de viabilité (Ouédraogo et al., 2003).

Si pour la germination des graines, le prétraitement a produit un effet néfaste et le substrat pratiquement pas d'effet, il semble qu'il n'en est pas de même pour la croissance des plantules issues des graines. En effet, sur tous les types de substrats, les plantules issues des graines traitées au feu ont montré les croissances les plus significatives pendant les trois premiers mois. Elles sont suivies

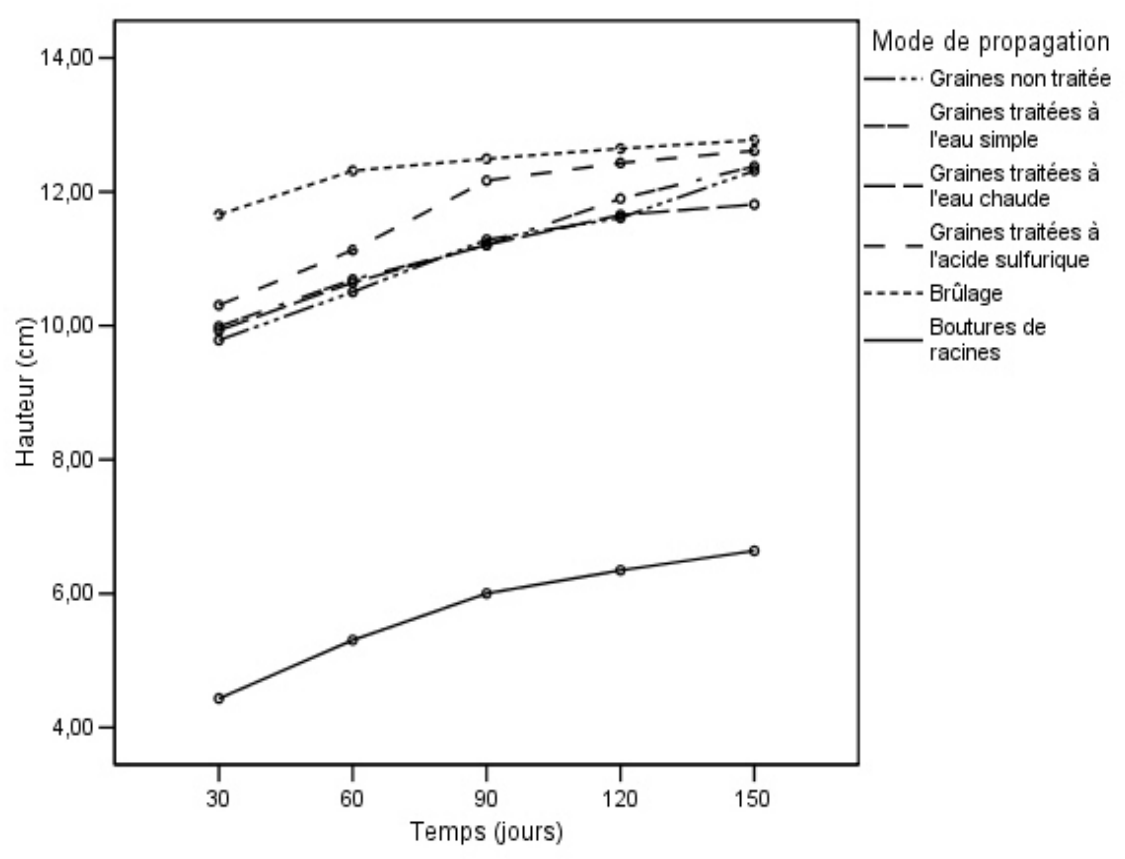

Figure 5. Comparaison de la croissance des plantules issues des graines et des pousses issues des boutures de racines. 
par les plantules issues des graines traitées à l'acide sulfurique. Après trois mois, la même tendance est maintenue sur les sols ferrugineux sablonneux, ferralitique et latéritique. Sur le sol ferrugineux argileux par contre entre quatre et cinq mois, c'est plutôt les plantules issues des graines non traitées qui montrent les meilleures hauteurs. Elles sont suivies des plantules issues du traitement au feu et du traitement à l'acide sulfurique. Ainsi, sur les sols ferrugineux argileux, on n'a peut-être pas besoin de prétraitement des graines pour stimuler la croissance en hauteur des plantules. Les croissances les moins fortes sont obtenues pour les plantules issues des graines traitées à l'eau simple et à l'eau chaude pour tous les types de sol.

Ces effets possibles du prétraitement sur le développement des plantules suggèrent que le prétraitement pourrait influer sur l'utilisation des réserves de la graine par les plantules, favorisant une croissance plus rapide, une vigueur plus grande et donc une meilleure chance d'établissement durant les premiers mois. En effet, la croissance et la vigueur des plantules dépendent de la disponibilité des réserves nutritives que la graine contient. Ainsi, par exemple, les semences les plus grosses ou lourdes génèrent généralement des plantules plus vigoureuses qui croissent et résistent mieux aux aléas du milieu (Khan et al., 1999; Baraloto et al., 2005; Bladé et Vallejo, 2008; Du et Huang, 2008). Un autre processus par lequel le prétraitement pourrait influer sur le développement des plantules est le raccourcissement du temps d'émergence de la plantule par élimination des possibles obstacles mécaniques au développement de la plantule (Du et Huang, 2008). Toutefois, des investigations complémentaires devraient être faites par d'autres essais et sur d'autres espèces pour confirmer ces relations entre le prétraitement et le développement des plantules.

De façon pratique, ces tendances suggèrent que, pour maximiser le nombre de plants et la croissance des plantules sur les cinq premiers mois, on peut utiliser des graines non traitées sur substrat argileux en pépinières ou en semis direct dans une zone où prédominent les sols argileux. Par contre, pour un autre type de sol ou de substrat comme le sol ferralitique, ferrugineux sablonneux ou latéritique, les graines peuvent être préalablement traitées pour stimuler la croissance des plantules. Mais il faudra tenir compte des pertes importantes à la germination dans l'estimation de la densité de semis ou du nombre de plants désirés pour les pépinières. Une solution intermédiaire pour avoir un taux de germination et une croissance des plantules relativement bonnes serait de tremper les graines dans une solution d'acide sulfurique.

Ces résultats expliquent aussi les observations faites pendant l'inventaire forestier national de 2007 au Bénin, qui a montré que la régénération naturelle de $D$. oliveri dans le domaine classé et protégé est presque nulle (IFN-PBF2, 2007). En effet, les peuplements de $D$. oliveri sont généralement situés dans des formations végétales à caractère savanicole. Les savanes sont très menacées par les feux de végétation qui sont allumés pendant la période de maturation des semences de l'espèce. Les effets néfastes du feu sur la germination des graines combinés avec la forte pression des prédateurs et des parasites, expliqueraient la faible régénération naturelle observée. Un phénomène similaire a été observé dans un essai de régénération artificielle par semis direct dans la zone de Benega à $160 \mathrm{~km}$ de Ouagadougou par Ouédraogo et al. (2003) qui ont obtenu un taux de survie de plantules de $D$. oliveri de $3,7 \%$ à un an, après un taux de levée initial $70 \%$. Les facteurs de stress étaient essentiellement le feu, les prédateurs et le piétinement par le bétail (Ouédraogo et al., 2003).

Concernant la multiplication végétative artificielle, les résultats ont montré que les boutures de tiges sont inappropriées pour reproduire l'espèce. Les pousses issues des bourgeons de ces boutures n'ont pas été viables car elles ont dégénéré très rapidement en l'intervalle de 30 jours et sont toutes mortes. Par contre, les boutures de racines ont été viables avec des taux de réussite de l'ordre de $66 \%$ et une croissance constante des jeunes pousses jusqu'à cinq mois après le début du bouturage. Il semble que, bien que l'enracinement n'ait pas été directement observé, les boutures de tiges n'auraient pas développé des racines pour leur survie, ce qui 
a fait qu' après l'épuisement des réserves au bout d'un mois, toutes les pousses ont dégénéré. Par contre, les boutures de racines ont probablement développé des racines adventives, ce qui explique la survie et la croissance des pousses sur plus de cinq mois.

La comparaison de la multiplication par graines et de la multiplication par boutures de racines du point de vue des taux de réussite suggère que le taux de réussite des boutures de racines est bien supérieur au taux de germination des graines traitées au feu, mais inférieur au taux de germination des graines non traitées ou traitées à l'acide sulfurique. Du point de vue de la croissance, les pousses issues des boutures de racines croissent beaucoup moins vite que tous les types de graines traitées. En somme, du point de vue de la croissance des jeunes pousses en pépinière, les boutures de racines ne présentent pas d'avantage par rapport au semis de graines.

Dans tous les cas, la bonne aptitude à la germination et à la conservation en milieu paysan des graines de $D$. oliveri (Ouedraogo et al., 2003) et la croissance relativement bonne des plantules sont autant d'avantages à l'actif de la multiplication par semis.

Toutefois, il convient de mener des essais sur la survie des plants issus des différents types de matériel de reproduction face à divers types de stress comme le feu, la sécheresse, le broutage, le piétinement, etc. Cette aptitude à résister au stress est déterminante dans la réussite de toute initiative de restauration.

\section{Conclusion}

En conclusion, cette étude a montré que les graines de $D$. oliveri ne présentent pas de problèmes de dormance et l'espèce peut être reproduite artificiellement par semis de graines mûres. Elle peut aussi être reproduite par boutures de racines. Toutefois, le semis présente l'avantage d'un meilleur taux de germination et d'une croissance plus rapide des plantules par rapport aux boutures de racines et il s'avère nécessaire de faire des investigations sur la survie des plants issus de chaque type de matériel végétal en milieu naturel face aux divers aléas pour choisir l'option la plus viable pour les programmes d'enrichissement ou d'installation de plantation. Par ailleurs, l'étude a aussi montré que, le prétraitement germinatif des graines, bien qu'ayant un effet néfaste sur la germination, pourrait bien stimuler la croissance des plantules.

\section{REFERENCES}

Ahmadua A, Zezib AU, Yarob AH. 2007. Anti-diarrheal activity of the leaf extracts of Daniellia oliveri Hutch and Dalz (Fabaceae) and Ficus sycomorus Miq (Moraceae). Afr. J. Tradit. Compliment. Altern. Med., 4(4): 524 - 528.

Arbonier M. 2000. Arbres et Arbustes et Lianes des Zones Sèches d'Afrique de l'Ouest. CIRAD, MNHN, UICP: Paris.

Aronson J, Floret C, LeFloc'h E, Ovalle C, Pontanier R. 1993. Restoration and rehabilitation of degraded ecosystems in arid and semi-arid lands. I. A view from the South. Restoration Ecology, 1: 8-17.

Baraloto C, Forget P-M, Goldberg DE. 2005. Seed mass, seedling size and neotropical tree seedling establishment. J. Ecol., 93: 1156-1166.

Bellefontaine R. 1997. Synthèse des espèces du domaine sahélien et soudanien qui se multiplient par voie végétative. In Fonctionnement et Gestion des Ecosystèmes Forestiers Contractés Sahéliens, d'Herbès JM, Amboula JMK, Peltier R (eds). John-Libbey Eurotext: Paris; 95-104.

Bellefontaine R, Gaston A, Petrucci Y. 2000. Management of natural forests of dry tropical zones. FAO Conservation Guide, 32: 69-166.

Bellefontaine R, Monteuuis O. 2002. Le drageonnage des arbres hors forêt: un moyen pour revégétaliser partiellement les zones arides et semi-arides sahéliennes? In Multiplication végétative des ligneux forestiers, fruitiers et ornementaux, Verger M (ed). Cirad-Inra: Montpellier; 135-148.

Bellefontaine R, Sabir M, Kokou K, Guinko S, Saadou M, Ichaou A, Hatem C, Bationo BA, Karim S, Logbo J. 2003. Revégétalisation, une Quatrième voie : la Propagation Végétative Naturelle. Mémoire Volontaire. XIIème Congrès Forestier Mondial, Septembre 2003, Congrès Forestier Mondial, Montréal, p. 8. 
Bellefontaine R. 2005. Régénération naturelle à faible coût dans le cadre de l'aménagement forestier en zones tropicales sèches en Afrique. Rev. Sci. Environ., 6(2) : 1-15.

Blade' C, Vallejo VR. 2008. Seed mass effects on performance of Pinus halepensis Mill. seedlings sown after fire. For. Ecol. Manag., 255: 2362-2372.

Cuny P, Sanogo S, Sommer N. 1997. Arbres du Domaine Soudanien : leurs Usages et leur Multiplication. Imprimerie COLOR: Bamako.

Du Y, Huang Z. 2008. Effects of seed mass and emergence time on seedling performance in Castanopsis chinensis. For. Ecol. Manag., 255: 2495-2501.

FAO. 2001a. Global Forest resources assessment 2000, Main report. FAO Forestry Paper 140, FAO, Rome, pp.1-16.

FAO. 2001b. State of Forest Genetic Resources in Sahelian and NorthSudanian Africa \& Sub-Regional Action Plan for their Conservation and Sustainable Use. Working Paper FGR/2E, Forest Resources Division FAO, Rome.

Ffolliott PF, Thames JL. 1983. Collection, handling, storage and pre-treatment of Prosopis seeds in Latin America. FAO, Rome, p. 52.

Geny P, Waechter P, Yatchinovsky A. 1992, Environnement et Développement Rural: Guide de Gestion des Ressources Naturelles. Frison-Roche: Paris.

Harviel A, Bellefontaine R, Boly O. 2006. Aptitude à la multiplication végétative de huit espèces forestières d'intérêt au Burkina Faso. Bois. For. Trop., 286(2): 40-50.
IFN-PBF2. 2007. Présentation des Résultats de l'Inventaire Forestier National. MAPS-DFS-CENATEL: Cotonou ; p.28.

Jegede IA, Nwinyi FC, Muazzam I, Akumka D, Njan A, Shok M. 2006. Micromorphological, anti-nociceptive and anti-inflammatory investigations of stem bark of Daniellia oliveri. Afr. J. Biotechnol., 5 (10): 930-935.

Khan ML, Bhuyan P, Shankar U, Todaria NP. 1999. Seed germination and seedling fitness in Mesua ferrea L. in relation to fruit size and seed number per fruit. Acta Oecologica , 20(6): 599-606.

Lykke AM. 2000. Local perceptions of vegetation change priorities for conservation of woody savanna vegetation in Senegal. . J. Environ. Manag., 59: 107 - 120.

Ouédraogo LG, Sary H, Yameogo-Gamene CS, Neya O. 2003. A Participatory Approach for Conservation of Forest Genetic Resources. In Conserving Biodiversity in Arid Regions: Best Practices in Developing Nations, Lemons J, Victor R, Schaffer D, (eds). Kluwer Academic Publishers; 63-72.

Robertson B. 2002. Opportunities for Growing Tree Crops in the Top End of the NT. Agnote, 23: 1-4.

Sokpon N, Ouisavi C. 2004. Gestion des plantations de Khaya senegalensis au Bénin. Bois. For. Trop., 279(1): 37 - 46.

Soto Flandez M. 1995. Dry forest silviculture in the Sudano-Sahelian region: Burkina Faso's experience. Unasylva, 181(2): 1317.

Zar JH. 1999. Biostatistical Analysis (4 ${ }^{\text {th }}$ edn) Prentice Hall: Upper Saddle River, New Jersey. 